\title{
Survival analysis following microwave ablation or surgical resection in patients with hepatocellular carcinoma conforming to the Milan criteria
}

\author{
QUAN SUN ${ }^{1-5}$, JINGXIANG SHI ${ }^{1-5}$, CHAOYI REN ${ }^{1-5}$, ZHI DU $^{1-5}$, GUIMING SHU $^{1-5}$ and YIJUN WANG ${ }^{1-5}$ \\ ${ }^{1}$ Department of Hepatobiliary Surgery, Tianjin Third Central Hospital; ${ }^{2}$ Tianjin Key Laboratory of Artificial Cells; \\ ${ }^{3}$ Artificial Cell Engineering Technology Research Center of The Public Health Ministry; ${ }^{4}$ Department of Hepatobiliary \\ Surgery, Nankai University Affiliated Third Central Hospital; ${ }^{5}$ Department of Hepatobiliary Surgery, \\ The Third Central Clinical College of Tianjin Medical University, Tianjin 300170, P.R. China
}

Received September 4, 2019; Accepted March 2, 2020

DOI: 10.3892/ol.2020.11529

\begin{abstract}
The aim of the present study was to compare the efficacy of microwave ablation (MWA) and surgical resection (RES) for the treatment of hepatocellular carcinoma (HCC) conforming to the Milan criteria and the associated short- and long-term survival rates. The baseline characteristics were obtained from 231 patients with $\mathrm{HCC}$ who met the Milan criteria. To compare the mortality rates between groups, survival analysis was conducted using the Kaplan-Meier method and the log-rank test. The factors associated with the survival rate were analyzed using Cox proportional hazard models. A total of 115 patients underwent RES, and 116 were treated with MWA. No significant differences were observed in the 1-, 3- and 5-year OS rates and the 1-year DFS rate between the two groups. The 7- and 10-year OS rates and the 3-, 5-, 7- and 10-year DFS rates of the RES group were significantly higher compared with those in the MWA group $(\mathrm{P}=0.004, \mathrm{P}=0.002, \mathrm{P}=0.003$ and $\mathrm{P}=0.002$, respectively). In addition, no marked differences were observed in the OS and DFS rates between the two groups of patients with solitary $\mathrm{HCC}$ lesions $\leq 3 \mathrm{~cm}(\mathrm{P}=0.066$ and $\mathrm{P}=0.056$ ) and in the OS of those with solitary lesions of $3-5 \mathrm{~cm}(\mathrm{P}=0.133)$; however the DFS of patients with single 3-5 cm HCC lesions in the RES group was notably higher compared with the MWA group $(\mathrm{P}=0.027)$. The Cox proportional hazard model revealed that age, hepatitis $\mathrm{B}$ and $C$ virus infection, tumor size, number, platelet count and the type of treatment intervention were risk factors affecting the survival and recurrence in patients with HCC. These
\end{abstract}

Correspondence to: Dr Yijun Wang, Department of Hepatobiliary Surgery, Tianjin Third Central Hospital, 83 Jintang Road, Hedong, Tianjin 300170, P.R. China

E-mail:wangyijuntj@163.com

Key words: hepatocellular carcinoma, surgical resection, microwave ablation, survival analysis results suggested that RES may provide superior survival benefits compared with MWA for patients with HCC who meet the Milan criteria.

\section{Introduction}

Primary liver cancer (PLC) refers to malignancies originating from hepatocytes and the bile duct epithelium (1). In 2018, PLC was the sixth most common type of cancer worldwide, after lung, breast, colorectal, prostate and gastric cancer (2). However, the poor prognosis of patients with PLC makes it the second leading cause of cancer-associated death worldwide (3). Contrary to the steady or declining trend of malignancies such as lung, breast and colon cancer, the incidence and mortality rates of PLC have increased rapidly in the past decade, and the Chinese population accounts for $\sim 50 \%$ of all global cases and deaths (4). In China, the incidence and mortality rates of PLC are 2,871/100,000 and 2,604/100,000 individuals, respectively, making it the fourth most common cancer type and the second leading cause of cancer-related death (5). The three different pathological types of PLC, hepatocellular carcinoma (HCC), intrahepatic cholangiocarcinoma (ICC) and mixed type HCC-ICC, differ in their pathogenesis, biological behavior, histological morphology, treatment methods and prognosis; $>85 \%$ of PLC cases are patients with $\mathrm{HCC}$ (6). HCC is responsible for $5 \%$ of all malignant tumors in humans and is the third leading cause of cancer-related death, second only to lung cancer and gastric cancer (1). HCC primarily occurs in chronic inflammatory environments (7). The majority of cases of HCC develop in the presence of advanced chronic liver disease associated with chronic hepatitis $\mathrm{B}$ virus (HBV) or hepatitis $\mathrm{C}$ virus (HCV) infection and alcoholism (8). According to the European Association for the Study of the Liver (EASL), the European Organization for Research and Treatment of Cancer and the American Association for the Study of Liver Diseases Guidelines, liver transplantation, radiofrequency ablation (RFA) and hepatectomy are the recommended treatments for HCC (9-11). Compared with other types of 
cancer, which are primarily treated by surgery, radiotherapy and chemotherapy, local treatment of HCC is widely used for therapeutic (ablation or surgery) and palliative (arterial chemoembolization) intentions (10). Microwave ablation (MWA) is a type of therapy that uses imaging technology to guide a microwave needle, which directly destroys tumor cells in the local area (12). It has advantages including reduced damage, significant short-term effects and wide indications compared with surgery, and is widely used in the clinic (13). However, a limited number of studies have been conducted on the comparison between the efficacy of MWA and surgical resection (RES). Our previous study found that the DFS rate of MWA under 3 years is lower compared with RES for HCC conforming to Milan criteria (14). The purpose of the present study was to compare the efficacy of MWA and RES treatments in patients with HCC within the Milan criteria, to analyze the impact of these two treatment types on overall survival (OS), DFS and recurrence, and to compare the effects of the two treatments on short-, medium- and long-term survival.

\section{Materials and methods}

Patients and sample collection. The records of the patients with HCC admitted to the Tianjin Third Central Hospital (Tianjin, China) between January 2004 and December 2012 were retrospectively analyzed. These patients were diagnosed based on cytohistological evidence or the diagnostic criteria of the EASL (10). In total, 231 patients initially treated with MWA or RES were selected.

The inclusion criteria were as follows: i) Meeting the Milan criteria, which are a single $\mathrm{HCC} \leq 5 \mathrm{~cm}$ or $\leq 3$ nodules of $<3 \mathrm{~cm}$ each; ii) no extrahepatic metastasis or notable vascular invasion; iii) liver function of Child-Pugh Class A or B; iv) no previous or simultaneous malignancies; and v) no previous treatment for HCC. The exclusion criteria were: i) Patients at Child-Pugh Class $\mathrm{C}$ or evidence of hepatic decompensation, including refractory ascites, esophageal or gastric variceal bleeding, or hepatic encephalopathy; ii) patients with severe coagulation disorders (platelet count $<50 \times 10^{9}$ cells/l or prothrombin time prolongation $>5 \mathrm{sec}$ ); and iii) patients who preferred liver transplantation $(9,14)$. The present study was approved by the Ethics Committee of Tianjin Third Central Hospital, and informed consent was obtained from each participant.

Study design. The 231 patients were categorized into MWA $(n=116)$ and RES $(n=115)$ groups based on the therapeutic method. The modified Response Evaluation Criteria in Solid Tumors (15) was used to assess the treatment response of the patients following MWA or RES.

MWA was performed at 2,450 MHz using a Forsea MTC-3 microwave therapeutic apparatus (Qinghai Microwave \& Electronic Research Institute). Lidocaine (2\%) was used for local anesthesia (Hubei Tianyao Pharmaceutical Co. Ltd.) and intravenous anesthesia included propofol (CordenPharma International) and fentanyl (Yichang Renfu Pharmaceuticals Co., Ltd.). After anesthesia was achieved, a 15-cm 14-gauge unipolar cooled-shaft antenna with an output power of 60-80 W was inserted into the center of the tumor. The ablation process was continuously guided and monitored using the
Philips IU-22 (Philips Medical Systems, Inc.) and the Aloka SSD 5000 (Hitachi-Aloka Medical, Ltd.) ultrasound systems with 1-5 MHz convex array probes. The number of ablation repetitions depended on the number, location, shape and coagulation function of the tumor. Ablation was completed when the tumor and a surrounding 1-cm safety margin were filled with hyperechoic microbubbles. MWA was used again as salvage treatment in patients with incomplete tumor ablation. To prevent bleeding and needle track implantation, the needle track was coagulated after completion of MWA. All MWA procedures were completed by ultrasound interventional doctors with $>5$ years of experience. All complications and adverse reactions were appropriately treated before the patient was discharged. Since the complications and adverse reactions occurred in only a few patients, the conditions of statistical analysis were not met and the analysis for the correlation between adverse reactions and survival was not conducted.

For RES, the patients were decubitus for general anesthesia and suitable rooftop incision. All surgeries were performed at the Department of Hepatobiliary Surgery of Tianjin Third Central Hospital by doctors with $>10$ years of experience. A margin of $\geq 1 \mathrm{~cm}$ was reserved for tumor resection. To avoid non-R0 resection, all surgeries were routinely performed with intraoperative ultrasonography, including estimating the number, size, location and blood supply of the tumor. Liver anatomy was assessed using a CUSA $^{\circledR}$ surgical system (Integra Life Sciences). Hepatic portal occlusion (Pringle Technologies, Inc.) was routinely applied, with blocking for $15 \mathrm{~min}$ and releasing for $5 \mathrm{~min}$. All surgical specimens were pathologically examined, and resection of the tumor without a margin was considered as R0 resection (7). All patients were appropriately treated after surgery, and their liver functions were close to normal prior to discharge.

Follow-up. To assess the effectiveness of treatment, an enhanced CT scan was routinely performed 1 month after the patient was treated; all patients were followed up. Serum $\alpha$-fetoprotein (AFP) levels were assessed and ultrasonography was performed every 3 months, and an enhanced CT scan was performed every 6 months. Local recurrence was defined as a CT result that revealed an abnormally enhanced area around the ablation lesion or local margin during follow-up. Intrahepatic recurrence was defined as neoplastic foci that occurred in the liver, but away from the ablation site or in the resected liver segment. Extrahepatic recurrence was defined as extrahepatic metastasis $(13,16)$. The primary endpoints were OS and DFS. The overall recurrence rate was also compared, and the study was completed by January 2018. No value was censored, all data were included in this study.

Statistical analysis. The data were statistically analyzed using SPSS software 21.0 (IBM Corp.), and the Student's t-test was applied to continuous variables. The data were presented as mean \pm SD. For categorical variables, the $\chi^{2}$ and Fisher's exact tests were used. The OS, DFS and overall recurrence curves were generated using the Kaplan-Meier method and compared with the log-rank test. Univariate and multivariate analyses of OS or DFS were performed using the Cox risk scale model. 
Table I. Baseline clinical characteristics of patients with HCC conforming to the Milan Criteria.

\begin{tabular}{|c|c|c|c|}
\hline Variable & MWA (n=116) & $\operatorname{RES}(n=115)$ & P-value \\
\hline Age, years mean $\pm \mathrm{SD}$ & $57.474 \pm 9.614$ & $54.461 \pm 10.366$ & 0.647 \\
\hline $\operatorname{Sex}(M / F), n$ & $92 / 24$ & $93 / 22$ & 0.767 \\
\hline $\mathrm{HBV} / \mathrm{HCV} / \mathrm{NBNC}, \mathrm{n}$ & $90 / 16 / 10$ & $104 / 9 / 2$ & $0.016^{\mathrm{a}}$ \\
\hline Cirrhosis (yes/no), $\mathrm{n}$ & $108 / 8$ & $108 / 7$ & 0.803 \\
\hline $\mathrm{BCLC}(0 / \mathrm{A} / \mathrm{B}), \mathrm{n}$ & $11 / 104 / 1$ & $7 / 108 / 0$ & 0.375 \\
\hline ALT, IU/l, median (range) & $35(9.0-99.0)$ & $40(14.0-93.0)$ & 0.145 \\
\hline AST, IU/1, median (range) & $28(4.0-109.0)$ & $32(5.0-95.0)$ & 0.581 \\
\hline Prothrombin time, sec mean \pm SD & $14.387 \pm 1.334$ & $14.215 \pm 1.390$ & 0.345 \\
\hline Total bilirubin, $\mu \mathrm{mol} / \mathrm{l}$, median (range) & $18.6(5.8-59.6)$ & $15.8(5.6-54.0)$ & 0.095 \\
\hline ALB, g/l, median (range) & $41.1(24.6-48.7)$ & $40.5(27.7-52.7)$ & 0.060 \\
\hline Ascites (absent/present), $\mathrm{n}$ & $100 / 16$ & $103 / 12$ & 0.434 \\
\hline Solitary tumor $(\leq 3 />3 \mathrm{~cm}), \mathrm{n}$ & $51 / 64$ & $41 / 73$ & 0.196 \\
\hline Tumor number $(1 / 2 / 3), \mathrm{n}$ & $94 / 17 / 5$ & $97 / 15 / 3$ & 0.716 \\
\hline $\operatorname{AFP}(<400 / \geq 400 \mathrm{ng} / \mathrm{ml}), \mathrm{n}$ & $86 / 23$ & $91 / 24$ & 0.966 \\
\hline Child-Pugh (A/B), n & $90 / 19$ & $99 / 16$ & 0.581 \\
\hline
\end{tabular}

${ }^{\mathrm{a}} \mathrm{P}<0.05$. HCC, hepatocellular carcinoma; HBV, hepatitis B virus; HCV, hepatitis C virus; BCLC, Barcelona Clinic Liver Cancer.

$\mathrm{P}<0.05$ was considered to indicate a statistically significant difference.

\section{Results}

Patients. No significant differences were observed in the baseline characteristics of patients between the two groups (Table I). Following treatment, the ablation rate of the MWA group was $99.14 \%$ (115/116), and incomplete ablation of the tumor was rectified by salvage MWA. In the RES group, all patients exhibited tumor-free resection margins of $\geq 1 \mathrm{~cm}$, which was determined during surgery by the naked eye or using ultrasonic guidance. All patients in the RES group received $\mathrm{R} 0$ resection (no residual tumor tissue was observed under the resection microscope); among them, the histological diagnoses were 35 well-differentiated, 62 moderately differentiated and 18 poorly differentiated cases of HCC.

Survival analysis. In the MWA group, the median follow-up time was $43.34 \pm 19.63$ months, and 82 patients died during the follow-up period. The causes of death included tumor progression (51/82), liver failure (11/82), gastrointestinal hemorrhage (6/82) and others not related to liver function/cancer (14/82). In the RES group, the median follow-up time was $50.36 \pm 24.28$ months; during this period, 65 patients died of tumor progression (40/65), liver failure (10/65), gastrointestinal hemorrhage (3/65) and other causes that were not related to liver function/cancer (12/65).

The mean OS times were 67.22 (95\% CI, 59.23-75.21) and 93.897 (95\% CI, 82.32-105.48) months, and the median OS times were 59.00 (95\% CI, 46.89-71.11) and 85.00 (95\% CI, 60.94-109.07) months in the MWA and RES groups, respectively. The OS rates for the MWA and RES groups were as follows: 1-year, 92.2 and 94.8\%; 3-year, 67.2 and 74.6\%; 5-year, 49.1 and 61.3\%; 7-year, 36.9 and 50.4\%; and 10-year, 20.5 and $41.1 \%$ (Table II). No significant differences were observed in the 1-, 3- and 5-year OS rates (Fig. 1A-C), although the 7- and 10-year OS rates were markedly different between the two groups $(\mathrm{P}=0.041$ and $\mathrm{P}=0.017$, respectively; Fig. 1D and E). The mean and median DFS times were 43.40 (95\% CI, 35.42-51.38) and 26.00 (95\% CI, 19.89-32.12) months in the MWA group, and 74.19 (95\% CI, 61.56-86.82) and 51.00 (95\% CI, 35.06-66.95) months in the RES group. The DFS rates for the MWA and RES groups were: 1-year, 73.8 and 90.4\%; 3-year, 41.8 and 60.2\%; 5-year, 25.8 and 43.9\%; 7-year, 22.6 and $41.4 \%$; and 10-year, 18.1 and $31.6 \%$ (Table II). Among these, no difference was observed in the 1-year DFS rate (Fig. 2A), but the 3-, 5-, 7- and 10-year DFS rates were significantly different between the MWA and RES groups $(\mathrm{P}=0.004$, $\mathrm{P}=0.002, \mathrm{P}=0.003$ and $\mathrm{P}=0.002$, respectively; Fig. 2B-E).

Subgroup analysis was performed on patients with solitary HCC nodules $\leq 3$ and $3-5 \mathrm{~cm}$. There were 39 and 36 cases of solitary HCC lesions $\leq 3 \mathrm{~cm}$ in the MWA and RES groups, respectively. The OS rates were: 1 -year, 97.4 and $100.0 \%$; 3-year, 76.9 and 83.3\%; 5-year, 64.1 and 75.0\%; 7-year, 50.3 and 62.7\%; and 10-year, 26.7 and 59.0\% (Table II). The DFS rates were: 1-year, 82.1 and $88.6 \%$; 3 -year, 51.1 and $72.7 \%$; 5-year, 41.5 and 57.6\%, 7-year, 31.6 and 46.5\%; and 10-year, 25.3 and $46.5 \%$ (Table II). No significant differences were observed in the OS and DFS rates between the two groups (Fig. 3A and B). For solitary HCC lesions between 3 and $5 \mathrm{~cm}$, there were 55 patients in the MWA group and 62 patients in the RES group. The 1-, 3-, 5-, 7-and 10-year OS rates in the MWA and RES groups were: 1-year, 90.9 and $93.5 \%$; 3-year, 65.5 and 74.2\%; 5-year, 47.3 and 61.0; 7-year, 34.6 and 48.2\%; and 10-year, 21.1 and $37.7 \%$ (Table II); no significant differences were observed between the two groups (Fig. 3C). The DFS rates of the MWA and RES groups were: 1-year, 68.4 and $83.6 \%$; 3-year, 41.1 and 58.8\%; 5-year, 23.1 and 41.7\%; 7-year, 23.1 and 32.8\%; and 10-year, 18.5 and $28.7 \%$ 
Table II. 1-, 3-, 5-, 7- and 10-year overall survival rate and disease-free survival rate of MWA, RES and their subgroups.

\begin{tabular}{|c|c|c|c|c|c|c|c|c|c|c|}
\hline \multirow[b]{2}{*}{ Group } & \multicolumn{5}{|c|}{ Overall survival rate $(\%)$} & \multicolumn{5}{|c|}{ Disease-free survival rate $(\%)$} \\
\hline & 1 year & 3 year & 5 year & 7 year & 10 year & 1 year & 3 year & 5 year & 7 year & 10 year \\
\hline MWA & 92.2 & 67.2 & 49.1 & 36.9 & 20.5 & 73.8 & 41.8 & 25.8 & 22.6 & 18.1 \\
\hline RES & 94.8 & 74.6 & 61.3 & 50.4 & 41.1 & 90.4 & 60.2 & 43.9 & 41.4 & 31.6 \\
\hline Single $\mathrm{HCC} \leq 3 \mathrm{~cm}$ in MWA & 97.4 & 76.9 & 64.1 & 50.3 & 26.7 & 82.1 & 51.1 & 41.5 & 31.6 & 25.3 \\
\hline Single $\mathrm{HCC} \leq 3 \mathrm{~cm}$ in RES & 100 & 83.3 & 75.0 & 62.7 & 59.0 & 88.6 & 72.7 & 57.6 & 46.5 & 46.5 \\
\hline Single HCC between $3-5 \mathrm{~cm}$ in MWA & 90.9 & 65.5 & 47.3 & 34.6 & 21.1 & 68.4 & 41.1 & 23.1 & 23.1 & 18.5 \\
\hline Single HCC between $3-5 \mathrm{~cm}$ in RES & 93.5 & 74.2 & 61.0 & 48.2 & 37.7 & 83.6 & 58.8 & 41.7 & 32.8 & 28.7 \\
\hline
\end{tabular}

MWA, microwave ablation; RES, surgical resection; HCC, hepatocellular carcinoma.

A

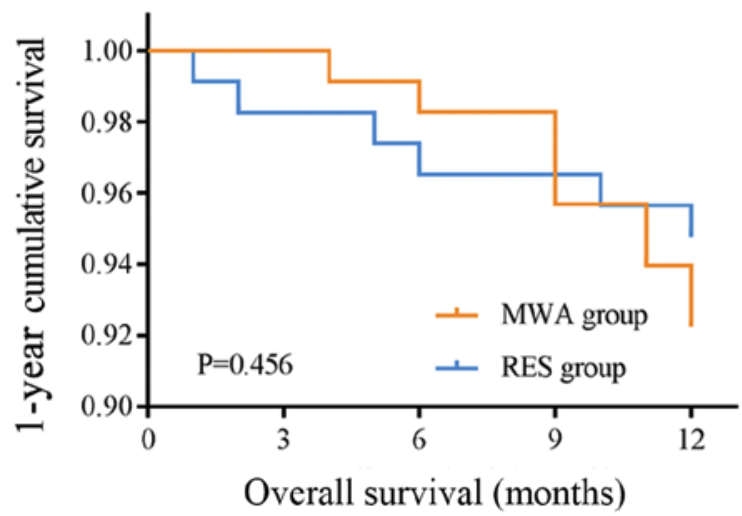

C

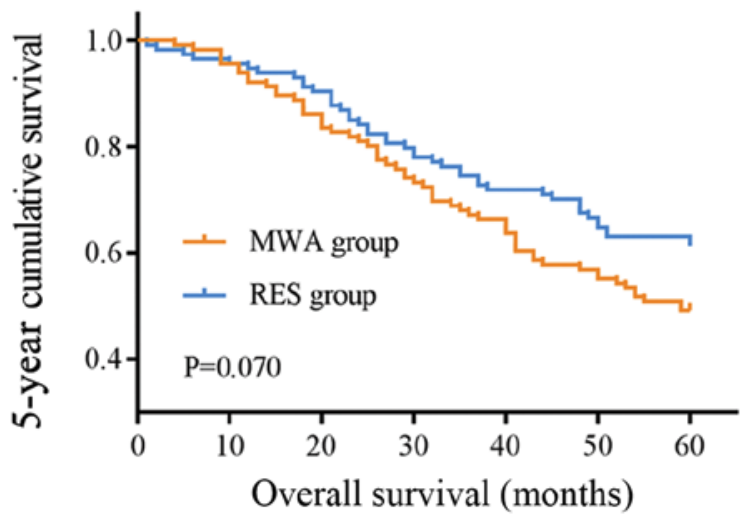

E

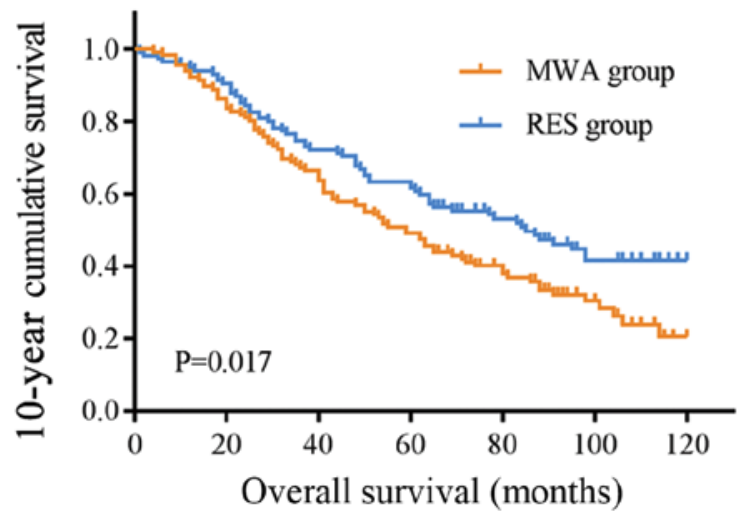

B

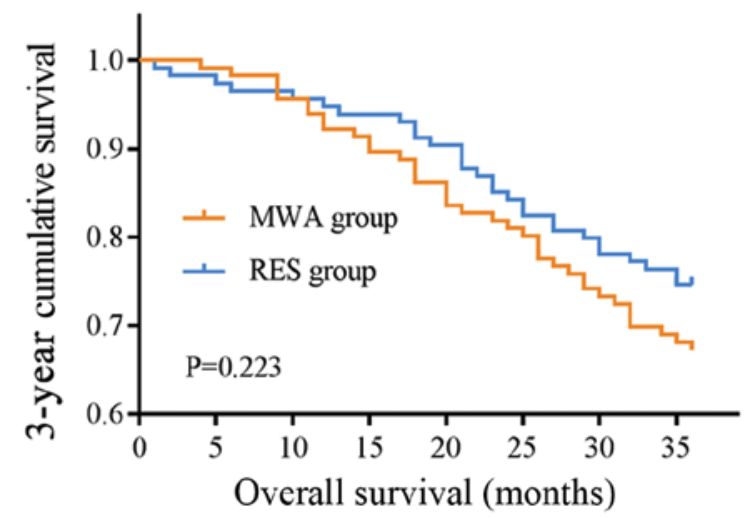

D

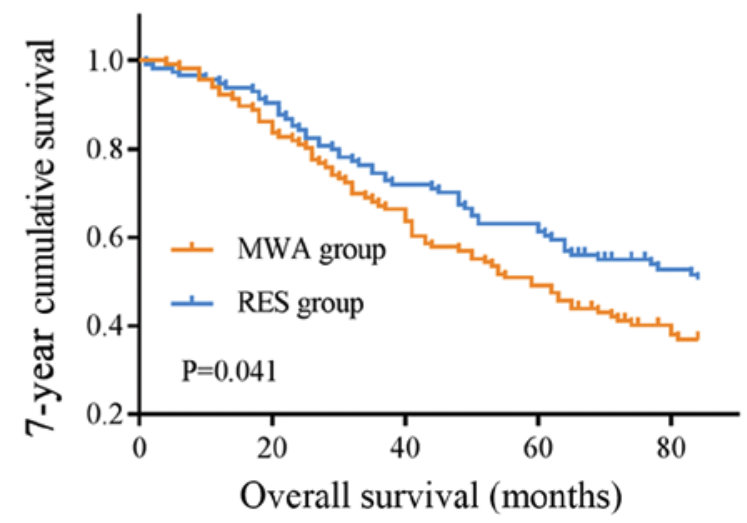

Figure 1. OS curves of patients who underwent MWA or RES. (A) 1-, (B) 3- and (C) 5-year OS rates were not different between the two groups. (D) 7- and (E) 10-year OS rates were higher in the RES group compared with those in the MWA group. OS, overall survival; MWA, microwave ablation; RES, surgical resection. 


\section{A}

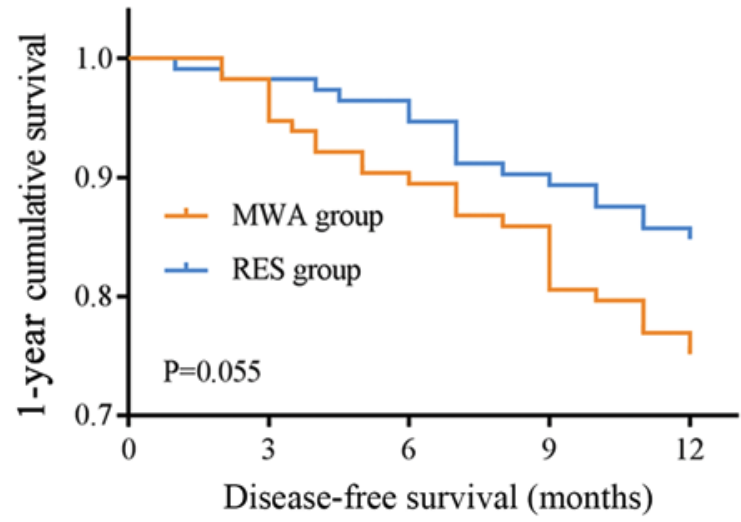

$\mathrm{C}$
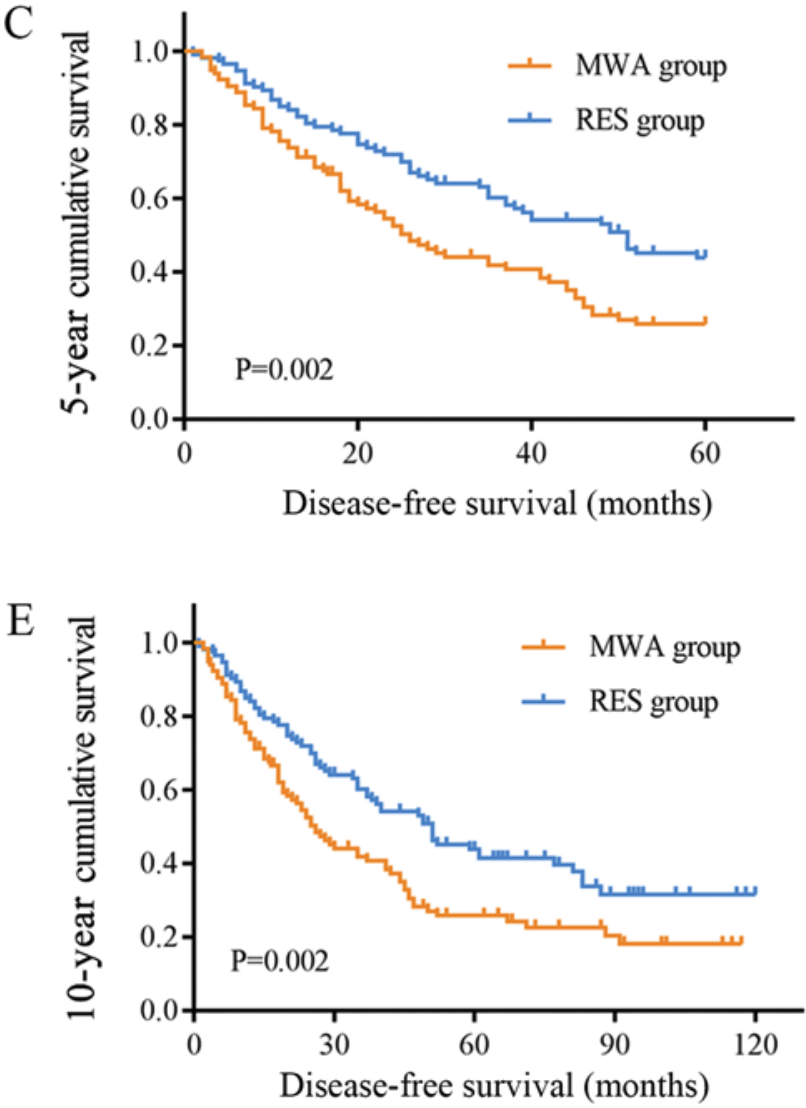

B

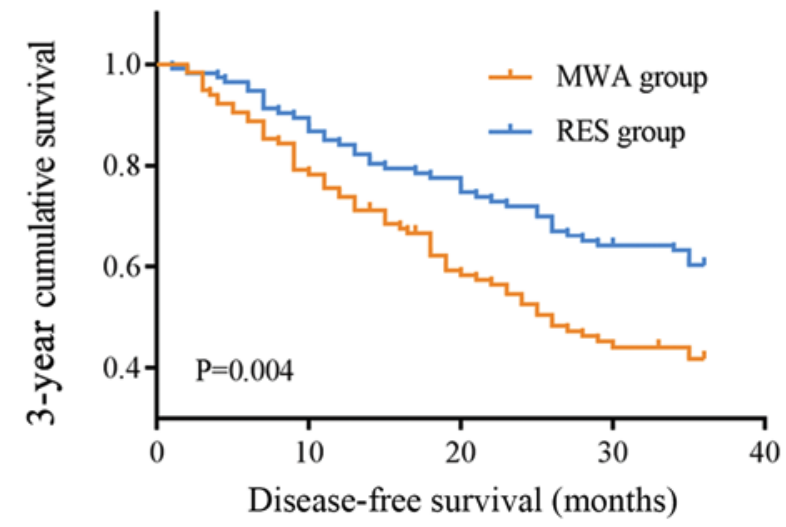

$\mathrm{D}$

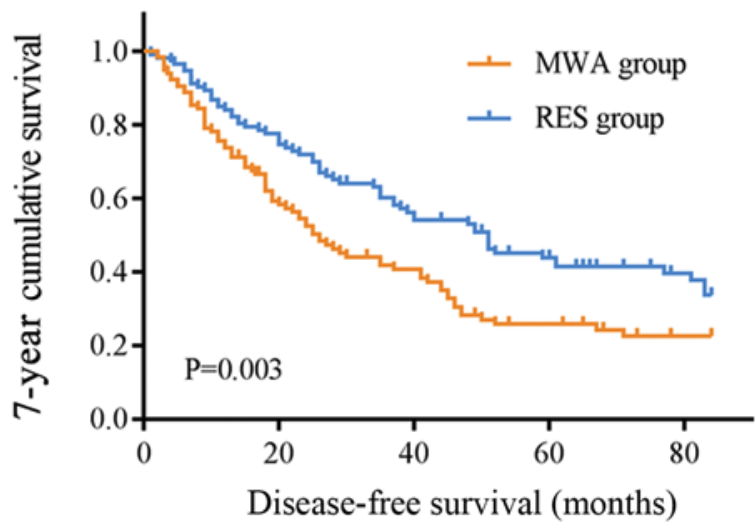

Figure 2. DFS curves of patients treated with MWA or RES. (A) 1-year DFS rates were not different between two groups. (B) 3-, (C) 5-, (D) 7- and (E) 10-year DFS rates in the RES group were significantly higher compared with those in the MWA group. DFS, disease-free survival; MWA, microwave ablation; RES, surgical resection.

(Table II), and the RES group exhibited a superior outcome compared with the MWA group ( $\mathrm{P}=0.027$, Fig. 3D).

Univariate and multivariate analysis. Among all variables, age $(\mathrm{P}=0.007)$, HBV $(\mathrm{P}=0.032)$ and $\mathrm{HCV}(\mathrm{P}=0.010)$ infection, platelet count $(\mathrm{P}=0.041)$, tumor number $(\mathrm{P}=0.001)$ and intervention type $(\mathrm{P}=0.019)$ were considered significant risk factors for OS. Following univariate analysis, variables with statistically significant differences were included in the Cox regression model. To avoid missing some important factors, the P-value was relaxed to 0.1 . A total of nine variables with $\mathrm{P}<0.1$ in the univariate analysis were included in Cox multivariate analysis, and tumor size $(\mathrm{P}=0.012)$, tumor number
$(\mathrm{P}=0.028)$ and intervention type $(\mathrm{P}=0.034)$ were considered to be significant risk factors for OS (Table III).

For univariate analysis, six variables [age $(\mathrm{P}=0.031), \mathrm{HBV}$ infection $(\mathrm{P}=0.039), \mathrm{HCV}$ infection $(\mathrm{P}=0.015)$, tumor size $(\mathrm{P}=0.048)$, tumor number $(\mathrm{P}=0.004)$ and intervention type $(\mathrm{P}=0.003)]$ were associated with DFS. Taking $\mathrm{P}<0.1$ in the univariate analysis as the standard, 10 variables were introduced into Cox multivariate analysis, the results of which revealed that tumor size $(\mathrm{P}=0.010)$ and intervention type $(\mathrm{P}=0.007)$ were associated with DFS (Table IV).

Recurrence analysis. At the end of the follow-up, recurrence had occurred in 89 patients in the MWA group and 80 patients 
Table III. Univariate and multivariate analysis of relative factors for overall survival.

\begin{tabular}{|c|c|c|c|c|c|}
\hline \multirow[b]{2}{*}{ Variable } & \multirow[b]{2}{*}{ Subgroup } & \multicolumn{2}{|c|}{ Univariate analysis } & \multicolumn{2}{|c|}{ Multivariate analysis } \\
\hline & & HR & P-value & $\mathrm{HR}$ & P-value \\
\hline Sex & Male vs. female & 0.897 & 0.612 & & \\
\hline Age, years & $\leq 65$ vs. $>65$ & 1.747 & $0.007^{\mathrm{a}}$ & 1.515 & 0.071 \\
\hline HBV & Yes vs. no & 0.642 & $0.032^{\mathrm{a}}$ & 0.769 & 0.582 \\
\hline $\mathrm{HCV}$ & Yes vs. no & 1.812 & $0.010^{\mathrm{a}}$ & 0.922 & 0.876 \\
\hline Liver cirrhosis & Yes vs. no & 0.799 & 0.248 & & \\
\hline ALT, IU/1 & $\leq 40$ vs. $>40$ & 0.896 & 0.514 & & \\
\hline AST, IU/l & $\leq 40$ vs. $>40$ & 0.804 & 0.219 & & \\
\hline Total bilirubin, $\mu \mathrm{mol} / 1$ & $\leq 19$ vs. $>19$ & 1.222 & 0.234 & & \\
\hline Serum albumin, $g / 1$ & $\leq 35$ vs. $>35$ & 1.296 & 0.172 & & \\
\hline Prothrombin time, sec & $\leq 15$ vs. $>15$ & 0.731 & 0.082 & 0.948 & 0.795 \\
\hline Platelet count $10^{9}$ cells $/ 1$ & $\leq 100$ vs. $>100$ & 1.408 & $0.041^{\mathrm{a}}$ & 1.216 & 0.333 \\
\hline Ascites & Absent vs. present & 1.201 & 0.458 & & \\
\hline Child-Pugh & A vs. B & 1.473 & 0.070 & 1.28 & 0.277 \\
\hline $\mathrm{AFP}, \mathrm{ng} / \mathrm{ml}$ & $\leq 400$ vs. $>400$ & 1.105 & 0.636 & & \\
\hline Tumor size, $\mathrm{cm}$ & $\leq 3$ vs. $>3$ & 1.404 & 0.051 & 1.589 & $0.012^{\mathrm{a}}$ \\
\hline Tumor number & Single vs. multiple & 0.509 & $0.001^{\mathrm{a}}$ & 0.607 & $0.028^{\mathrm{a}}$ \\
\hline Intervention & RES vs. MWA & 0.673 & $0.019^{\mathrm{a}}$ & 0.687 & $0.034^{\mathrm{a}}$ \\
\hline
\end{tabular}

${ }^{\mathrm{a}} \mathrm{P}<0.05$. HR, hazard ratio; HBV, hepatitis B virus; $\mathrm{HCV}$, hepatitis $\mathrm{C}$ virus.

A Solitary $\mathrm{HCC} \leq 3 \mathrm{~cm}$

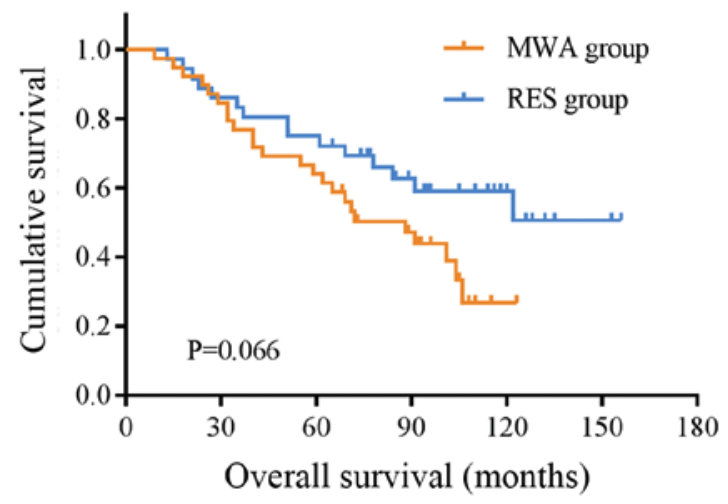

C Solitary $\mathrm{HCC}$ of $3-5 \mathrm{~cm}$

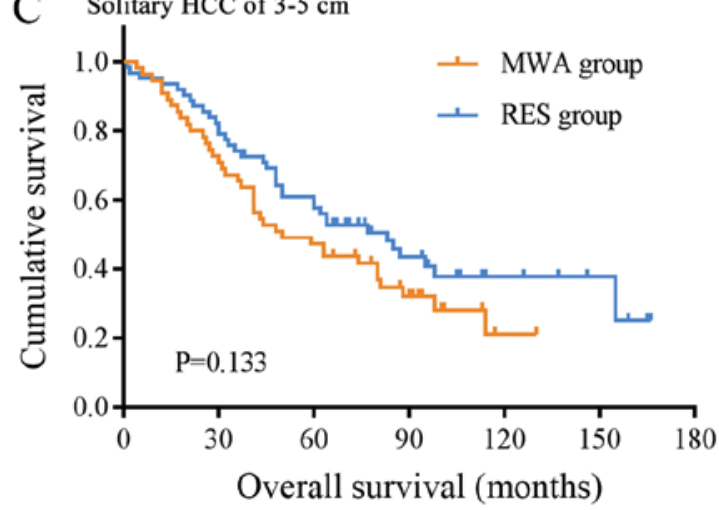

B Solitary $\mathrm{HCC} \leq 3 \mathrm{~cm}$

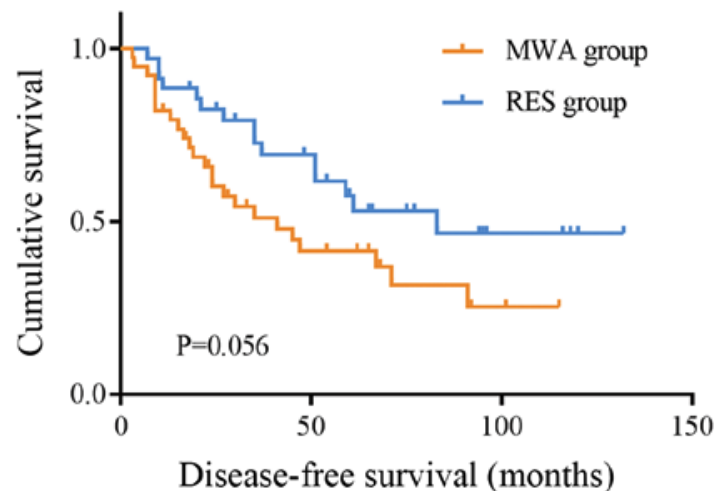

D Solitary HCC of $3-5 \mathrm{~cm}$

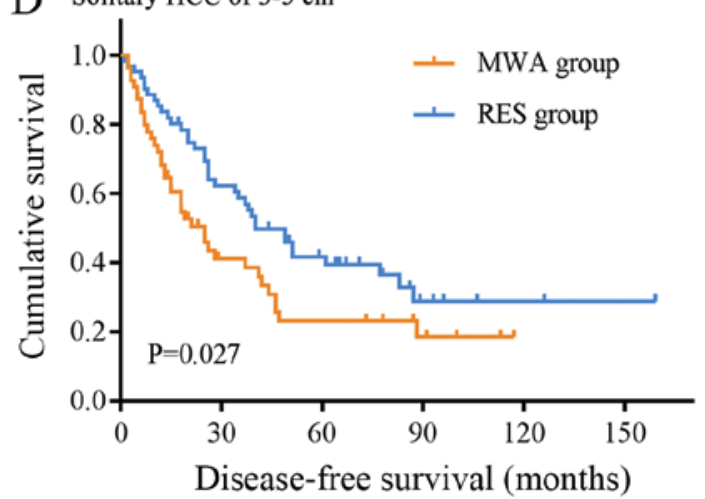

Figure 3. Subgroup analysis of OS and DFS of patients treated with MWA or RES. (A) OS time of patients with solitary HCC lesions $\leq 3 \mathrm{~cm}$. (B) DFS time of patients with solitary HCC lesions $\leq 3 \mathrm{~cm}$ and (C) OS time of patients with solitary HCC lesions of 3-5 cm were not significantly different between two groups. (D) DFS time of patients with solitary HCC lesions of 3-5 cm was longer in the RES group compared with that in the MWA group. OS, overall survival; DFS, disease-free survival; MWA, microwave ablation; RES, surgical resection; HCC, hepatocellular carcinoma. 
Table IV. Univariate and multivariate analysis of relative factors for disease-free survival.

\begin{tabular}{|c|c|c|c|c|c|}
\hline \multirow[b]{2}{*}{ Variable } & \multirow[b]{2}{*}{ Subgroup } & \multicolumn{2}{|c|}{ Univariate analysis } & \multicolumn{2}{|c|}{ Multivariate analysis } \\
\hline & & HR & P-value & HR & P-value \\
\hline Sex & Male vs. female & 0.804 & 0.308 & & \\
\hline Age, years & $\leq 65$ vs. $>65$ & 1.553 & $0.031^{\mathrm{a}}$ & 1.308 & 0.243 \\
\hline $\mathrm{HBV}$ & Yes vs. no & 0.655 & $0.039^{\mathrm{a}}$ & 0.641 & 0.355 \\
\hline $\mathrm{HCV}$ & Yes vs. no & 1.761 & $0.015^{\mathrm{a}}$ & 0.766 & 0.614 \\
\hline Liver cirrhosis & Yes vs. no & 0.582 & 0.162 & & \\
\hline ALT, IU/1 & $\leq 40$ vs. $>40$ & 0.994 & 0.972 & & \\
\hline AST, IU/1 & $\leq 40$ vs. $>40$ & 0.786 & 0.174 & & \\
\hline Total bilirubin, $\mu \mathrm{mol} / 1$ & $\leq 19$ vs. $>19$ & 1.164 & 0.367 & & \\
\hline Serum albumin, $\mathrm{g} / \mathrm{l}$ & $\leq 35$ vs. $>35$ & 1.398 & 0.078 & 1.083 & 0.705 \\
\hline Prothrombin time, sec & $\leq 15$ vs. $>15$ & 0.720 & 0.069 & 0.838 & 0.395 \\
\hline Platelet count $10^{9}$ cells $/ 1$ & $\leq 100$ vs. $>100$ & 1.325 & 0.093 & 1.101 & 0.640 \\
\hline Ascites & Absent vs. present & 1.171 & 0.522 & & \\
\hline Child-Pugh & A vs. B & 1.446 & 0.084 & 1.296 & 0.257 \\
\hline $\mathrm{AFP}, \mathrm{ng} / \mathrm{ml}$ & $\leq 400$ vs. $>400$ & 1.167 & 0.464 & & \\
\hline Tumor size, $\mathrm{cm}$ & $\leq 3$ vs. $>3$ & 1.409 & $0.048^{\mathrm{a}}$ & 1.621 & $0.010^{\mathrm{a}}$ \\
\hline Tumor number & Single vs. multiple & 0.557 & $0.004^{\mathrm{a}}$ & 0.650 & 0.061 \\
\hline Intervention & RES vs. MWA & 0.607 & $0.003^{\mathrm{a}}$ & 0.607 & $0.007^{\mathrm{a}}$ \\
\hline
\end{tabular}

${ }^{\text {a }}<0$.05. HR, hazard ratio; HBV, hepatitis B virus; $\mathrm{HCV}$, hepatitis $\mathrm{C}$ virus.
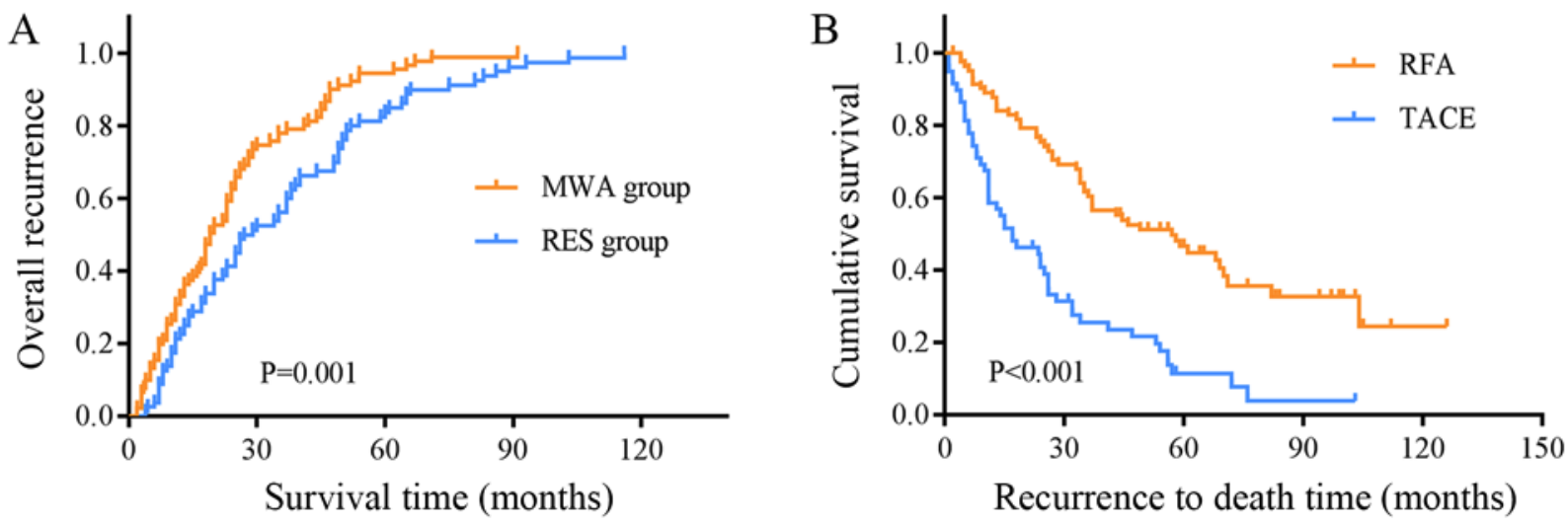

Figure 4. Recurrence curves of patients who underwent MWA or RES. (A) The overall recurrence rate in the MWA group was significantly higher compared with that in the RES group. (B) The mean survival time of patients with recurrence treated with RFA was different compared with that of patients treated TACE. MWA, microwave ablation; RES, surgical resection; RFA, radiofrequency ablation; TACE, transcatheter arterial chemoembolization.

in the RES group. The overall recurrence rates in the two groups were: 1-year, 5.3 and $1.8 \%$; 3 -year, 27.5 and $20.3 \%$; 5-year, 43.7 and 30.8\%; 7-year, 63.5 and 48.1\%; and 10-year, 93.7 and $72.9 \%$ (Fig. 4A). The overall recurrence rate in the MWA group was significantly higher compared with that in the RES group ( $\mathrm{P}=0.001$; Fig. $4 \mathrm{~A})$. The recurrence rate in the early stage (recurrence within 2 years) in the MWA group was higher compared with that in the RES group (57/89 vs. 34/80; $\mathrm{P}=0.003$; Table V). No significant difference was observed in the recurrence location between the two groups (Table V); however, the local recurrence rate in the MWA group was significantly higher compared with that in the RES group (12/116 vs. $4 / 115 ; \mathrm{P}=0.026)$.
Subgroup analysis revealed that the recurrence rate for solitary HCC lesions $\leq 3 \mathrm{~cm}$ and those of $3-5 \mathrm{~cm}$ did no differ between the two groups. In lesions between 3 and $5 \mathrm{~cm}$, the local recurrence rates of the MWA and the RES groups were markedly different (local/intrahepatic recurrence: $8 / 38$ vs. $1 / 48 ; \mathrm{P}=0.012$; Table $\mathrm{V}$ ), and the local recurrence rate was higher compared with the intrahepatic recurrence rate. By contrast, no difference was observed in the local recurrence rate between the two groups (4/34 vs. 2/22; Table V) in patients with solitary HCC lesions $\leq 3 \mathrm{~cm}$.

Among the 89 patients with recurrence in the MWA group, 50 received RFA, 2 received RES, 30 received transcatheter arterial chemoembolization (TACE), 2 received systemic 
Table V. Recurrence analysis and recurrence therapy of patients treated with MWA or RES.

\begin{tabular}{|c|c|c|c|}
\hline Variable & MWA (89/116) & RES (80/115) & P-value \\
\hline Single $\mathrm{HCC} \leq 3 \mathrm{~cm}$ & $29 / 38$ & $20 / 36$ & $0.050^{\mathrm{a}}$ \\
\hline Single HCC between $3-5 \mathrm{~cm}$ & $40 / 55$ & $45 / 62$ & 0.57 \\
\hline Early-stage recurrence ( $<2$ years $)$ & $57 / 89$ & $34 / 80$ & $0.003^{\mathrm{a}}$ \\
\hline \multicolumn{4}{|l|}{ Recurrence location } \\
\hline Local & 12 & 4 & \multirow[t]{3}{*}{0.062} \\
\hline Intrahepatic & 73 & 71 & \\
\hline Extrahepatic & 4 & 5 & \\
\hline \multicolumn{4}{|c|}{ Recurrence location for single $\mathrm{HCC} \leq 3 \mathrm{~cm}$} \\
\hline Local & 4 & 2 & \multirow[t]{2}{*}{0.572} \\
\hline Intrahepatic & 34 & 22 & \\
\hline \multicolumn{4}{|c|}{ Recurrence location for single $\mathrm{HCC}$ between $3-5 \mathrm{~cm}$} \\
\hline Local & 8 & 1 & \multirow[t]{2}{*}{$0.012^{\mathrm{a}}$} \\
\hline Intrahepatic & 38 & 48 & \\
\hline \multicolumn{4}{|l|}{ Recurrence treatment } \\
\hline RFA & 50 & 34 & \multirow[t]{3}{*}{0.153} \\
\hline TACE & 30 & 33 & \\
\hline Other & 9 & 13 & \\
\hline \multicolumn{4}{|c|}{ Recurrence treatment for single $\mathrm{HCC} \leq 3 \mathrm{~cm}$} \\
\hline RFA & 17 & 12 & \multirow[t]{3}{*}{0.598} \\
\hline TACE & 9 & 4 & \\
\hline Other & 3 & 4 & \\
\hline \multicolumn{4}{|c|}{ Recurrence treatment for single $\mathrm{HCC}$ between $3-5 \mathrm{~cm}$} \\
\hline RFA & 25 & 19 & \multirow[t]{3}{*}{0.159} \\
\hline TACE & 12 & 19 & \\
\hline Other & 3 & 7 & \\
\hline
\end{tabular}

${ }^{\mathrm{a}} \mathrm{P}<0.05$. MWA, microwave ablation; RES, surgical resection; HCC, hepatocellular carcinoma; RFA, radiofrequency ablation; TACE, transarterial chemoembolization.

chemotherapy, 2 received symptomatic treatment, 1 received supportive treatment and 2 did not receive treatment. Among the 80 patients with recurrence in the RES group, 37 underwent RFA, 3 underwent RES, 32 underwent TACE, 2 received systemic chemotherapy, 2 received symptomatic treatment, 1 received supportive treatment and 3 did not receive treatment. No significant differences were observed in the results of radical treatment (local ablation or RES) between the two groups (52/89 vs. 36/80), nor between all patients with recurrence receiving RFA and TACE (84/169 vs. 63/169). For all patients with recurrence, the mean survival time of patients with recurrent HCC treated with RFA was $63.55 \pm 5.43$ months (95\% CI, 52.92-74.19) and for those treated with TACE, it was 27.11 \pm 3.58 months (95\% CI, 20.09-34.12), revealing a distinct difference between the two treatment types $(\mathrm{P}<0.001$, Fig. 4B).

\section{Discussion}

HCC is one of the most prevalent types of cancer globally, but progress in the development of effective treatments for advanced disease has been limited (17). Due to its complexity (with early symptoms that are not obvious, usually with cirrhosis, recurrence, metastasis and heterogeneity after surgery), HCC is one of the most lethal malignant tumor types (18). The majority of patients are diagnosed in the late stages of disease and have missed the optimal surgical period; therefore, non-surgical resection has become the treatment of choice for advanced HCC $(19,20)$. Ablation therapy and TACE are common treatments for localized diseases. Ablation using alcohol, radiofrequency, microwave or cryoablation is considered a therapeutic option for surgical excision $(21,22)$. As well as RES and liver transplantation, RFA has been recognized as the first-line treatment for small HCCs $(<3 \mathrm{~cm})(23)$. However, multiple retrospective and prospective randomized controlled trials have demonstrated that there is no significant difference in survival between RFA and RES for the treatment of small hepatic lesions (24). By contrast, RES is a more clinically established method, with lower recurrence rates and prolonged DFS compared with RFA, and can be used to remove multiple lesions, satellite occlusions and tumor thrombi in the same liver segment (25). MWA therapy is a treatment method that has been developed in recent years; it is an effective treatment for liver cancer due to its minimal invasiveness, safety and wide range of indications for local 
tumor treatment (26). Compared with RFA, MWA provides a larger ablation range and higher intratumoral temperature and is less influenced by the heat sink effect (27). Therefore, MWA technology can theoretically achieve ideal local tumor control. Lucchina et al (28) reviewed six studies of MWA and RFA in the treatment of HCC; compared with RFA, the 1- and 3-year survival rates of patients treated with MWA were 89-100 and $49-80 \%$, respectively, with fewer postoperative complications.

In the present study, survival analysis revealed no differences in OS and DFS rates between the MWA and RES groups in the short-term ( $\leq 5$ years), which was consistent with our previous study (14). For long-term survival (7 and 10 years), both the OS and DFS rates of the RES group were significantly higher compared with those of the MWA group. In addition, the total and early recurrence rates in the MWA group were significantly higher compared with those in the RES group. In theory, RES has the advantage of providing improved local control of HCC, whereas MWA is limited by the location of the lesion. For isolated small liver cancers, $\geq 13 \%$ of cases may have small accessory tumors near the primary tumor that are not detected by imaging (29). In addition, MWA is also limited by the size of the lesion; for tumors $>3 \mathrm{~cm}$, MWA may result in insufficient ablation, and residual tumor tissue may potentially result in local recurrence (30). Moreover, RES is able to remove small tumor satellites (30), which may reduce intrahepatic recurrence rates compared with MWA.

Subgroup analysis in the present study indicated that no differences between the OS and DFS rates in the MWA and RES groups for patients with solitary $\mathrm{HCC}$ lesions $\leq 3 \mathrm{~cm}$, and in the OS rate for solitary HCCs $>3 \mathrm{~cm}$. By contrast, there was a significant difference in the DFS rate of patients with solitary HCC lesions $>3 \mathrm{~cm}$ between the two groups. The size of a tumor is an indication of its age; the longer the time in vivo, the greater the degree of microvascular invasion to the surrounding tissues, and the more the recurrence and survival rates are affected $(31,32)$. Lazzara et al $(33)$ have reported that a safety margin of $<1.0 \mathrm{~cm}$ is an independent factor for early recurrence of liver cancer. In the present study, RES resulted in a more adequate safety margin. Additionally, MWA produces a necrotic area of $\sim 4.8 \times 4 \times 4 \mathrm{~cm}$, providing a $1.0 \mathrm{~cm}$ safety margin for tumors $<3 \mathrm{~cm}$ in diameter (34), which may explain the similarity in the OS and DFS rates of patients with single HCC lesions $\leq 3 \mathrm{~cm}$ in the two groups in the present study. By contrast, single HCC lesions $>3 \mathrm{~cm}$ are commonly accompanied by microsatellite foci and vascular invasion, which are risk factors for survival and recurrence in HCC treated by RES, MWA or hepatic artery chemotherapy (35). The most important point in ablation therapy for medium- and large-sized HCC lesions is that the tissue coagulation area formed by a single energy output needs to be sufficiently large. In large tumors, even with multipoint ablation, it is still possible to retain a gap (i.e. residual cancer tissue) due to the small range of the single coagulation area and the incomplete overlap of the ablation foci (36). Therefore, the local recurrence rate is higher after ablation. In the present study, for multiple tumors, no differences were observed in OS and DFS rates between the MWA and RES groups. This was primarily due to the multicentric nature of HCC, and the fact that most patients had a history of chronic HBV infection and cirrhosis. Following surgical resection, these tumorous tissues were still present in the residual liver, resulting in a high risk of recurrence.

In the present study, age, $\mathrm{HBV}$ and $\mathrm{HCV}$ infection, tumor size, tumor number and intervention type were significantly associated with OS and DFS rates. Pompili et al (37) have reported that elderly patients are at a higher risk of death from extrahepatic diseases and are more likely to suffer from liver failure, as they may suffer a longer course of chronic liver disease. Studies have also suggested that HBV and HCV infections are the primary causes of HCC (38). Active HBV or HCV infection causes liver necrosis that may lead to gene mutations and promote recurrence and metastasis (39). Tumor size and number are the other two major factors that influence the postoperative metastasis and recurrence of HCC; lesions with a diameter $>5 \mathrm{~cm}$, multiple tumors and vascular or microvascular infiltration are frequently observed in tumor microsatellite foci far from the primary tumor, which reduces the possibility of radical resection and significantly increases the rate of recurrence (40). These results are in agreement with those of the present study.

There were several limitations to the current study. Since this was a single center retrospective analysis, the selection of patients may have been biased. For further investigation, prospective randomized controlled trials are being considered. The sample size was also limited and did not reveal statistical significance in certain comparisons. In addition, a number of patients were followed-up for $>10$ years, whereas others were only assessed for $\leq 5$ years; thus, further follow-up is required.

In summary, the present study indicated that for patients with HCC meeting the Milan criteria, MWA resulted in a higher tumor recurrence rate and lower DFS rate compared with those of patients treated with RES. For the short term, the effects of the two therapies were comparable, but the long-term survival rate was higher in the RES group. For patients with solitary HCC lesions $\leq 3 \mathrm{~cm}$, MWA and RES were equally effective, although for patients with solitary HCC lesions $>3 \mathrm{~cm}$, the DFS was longer after RES treatment. A multicenter, large-sample, prospective randomized controlled study is being considered for further investigation.

\section{Acknowledgements}

Not applicable.

\section{Funding}

This work was supported by the Program of Tianjin Science and Technology Development Plan (grant. no. 17YFZCSY01070).

\section{Availability of data and materials}

All data analyzed during this study are included in this published article.

\section{Authors' contributions}

QS made substantial contributions to the conception and design and wrote the manuscript. JS and CR acquired the data. ZD and GS analyzed and interpreted the data. YW contributed to conception and design of this study, reviewed 
the manuscript and gave final approval of the version to be published and agreed to be accountable for all aspects of the work. All authors read and approved the final manuscript.

\section{Ethics approval and consent to participate}

Informed consent was obtained from the participants. This study was approved by the Ethical Committee of Tianjin Third Central Hospital.

\section{Patient consent for publication}

Not applicable.

\section{Competing interests}

The authors declare that they have no competing interests.

\section{References}

1. Cross TJS and Evans JC (eds.): Transarterial embolization therapies in hepatocellular carcinoma: Principles of management: From mechanisms to management. In: Liver Cancers. Springer International Publishing Corp., Berlin, pp123-138, 2019.

2. Valery PC, Laversanne M, Clark PJ, Petrick JL, McGlynn KA and Bray F: Projections of primary liver cancer to 2030 in 30 countries worldwide. Hepatology 2: 600-611, 2018.

3. Cong WM, Bu H, Chen J, Dong H, Zhu YY, Feng LH and Chen J; Guideline Committee: Practice guidelines for the pathological diagnosis of primary liver cancer: 2015 update. World J Gastroenterol 22: 9279-9287, 2016.

4. Torre LA, Bray F, Siegel RL, Ferlay J, Lortet-Tieulent J and Jemal A: Global cancer statistics, 2012. CA Cancer J Clin 65: 87-108, 2015.

5. Chen WQ, Zheng RS and Zhang SW: Liver cancer incidence and mortality in China, 2009. Chin J Cancer 32: 162-169, 2013.

6. Bureau of Medical Administration, National Health and Family Planning Comission of the People's Republic of China: Diagnosis, management, and treatment of hepatocellular carcinoma (V2017). Zhonghua Gan Zang Bing Za Zhi 25: 886-895, 2017 (In Chinese).

7. Duffy AG, Ulahannan SV, Makorova-Rusher O, Rahma O, Wedemeyer H, Pratt D, Davis JL, Hughes MS, Heller T, ElGindi M, et al: Tremelimumab in combination with ablation in patients with advanced hepatocellular carcinoma. J Hepatol 66: 545-551, 2017.

8. Klein S and Dufour JF: Nonalcoholic fatty liver disease and hepatocellular carcinoma. Hepat Oncol 4: 83-98, 2017.

9. Boyer TD and Haskal ZJ; American Association for the Study of Liver Diseases: The role of transjugular intrahepatic portosystemic shunt (TIPS) in the management of portal hypertension: Update 2009. Hepatology 51: 306, 2010.

10. European Association for the Study of the Liver. Electronic address: easloffice@easloffice.eu; European Association for the Study of the Liver: EASL clinical practice guidelines: Management of hepatocellular carcinoma. J Hepatol 69: 182-236, 2018.

11. Fukuda H: European organization for research and treatment of cancer. Jpn J Clin Oncol 30: 169, 2000.

12. Zhou Y, Xu X, Ding J, Jing X, Wang F, Wang Y and Wang P: Dynamic changes of T-cell subsets and their relation with tumor recurrence after microwave ablation in patients with hepatocellular carcinoma. J Cancer Res Ther 14: 40-45, 2018.

13. Peng ZW, Lin XJ, Zhang YJ, Liang HH, Guo RP, Shi M and Chen MS: Radiofrequency ablation versus hepatic resection for the treatment of hepatocellular carcinomas $2 \mathrm{~cm}$ or smaller: A retrospective comparative study. Radiology 262: 1022-1033, 2012.

14. Shi J, Sun Q, Wang Y, Jing X, Ding J, Yuan Q, Ren C, Shan S, Wang Y and Du Z: Comparison of microwave ablation and surgical resection for treatment of hepatocellular carcinomas conforming to Milan criteria. J Gastroenterol Hepatol 29: $1500-1507,2014$.
15. Lencioni R and Llovet JM: Modified RECIST (mRECIST) assessment for hepatocellular carcinoma. Semin Liver Dis 1: 52-60, 2010.

16. Goldberg SN, Grassi CJ, Cardella JF, Charboneau JW, Dodd GD III, Dupuy DE, Gervais D, Gillams AR, Kane RA, Lee FT $\mathrm{Jr}$, et al: Image-guided tumor ablation: Standardization of terminology and reporting criteria. Radiology 235: 728-739, 2005.

17. Llovet JM and Hernandez-Gea V: Hepatocellular carcinoma: Reasons for phase III failure and novel perspectives on trial design. Clin Cancer Res 20: 2072-2079, 2014.

18. Li X, Li B, Li B, Guo T, Sun Z, Li X, Chen L, Chen W, Chen P, Mao Y and Zeng Y: ITIH4: Effective serum marker, early warning and diagnosis, hepatocellular carcinoma. Pathol Oncol Res 24: 663-670, 2018.

19. Bréchot C: Pathogenesis of hepatitis B virus-related hepatocellular carcinoma: Old and new paradigms. Gastroenterology 127 (5 Suppl 1): S56-S61, 2004.

20. El-Serag HB: Epidemiology of viral hepatitis and hepatocellular carcinoma. Gastroenterology 142: 1264-1273.e1, 2012.

21. Chen MS, Li JQ, Zheng Y, Guo RP, Liang HH, Zhang YQ, Lin XJ and Lan WY: A prospective randomized trial comparing percutaneous local ablative therapy and partial hepatectomy for small hepatocellular carcinoma. Ann Surg 243: 321-328, 2006.

22. Feng K, Yan J, Li X, Xia F, Ma K, Wang S, Bie P and Dong J: A randomized controlled trial of radiofrequency ablation and surgical resection in the treatment of small hepatocellular carcinoma. J Hepatol 57: 794-802, 2012.

23. Thandassery RB, Goenka U and Goenka MK: Role of local ablative therapy for hepatocellular carcinoma. J Clin Exp Hepatol 4 (Suppl 3): S104-S111, 2014.

24. Yan X, Fu BM, Tang B, et al: Progress in clinical research of radiofrequency ablation for primary liver cancer. J Hepato Surg 1: 87-89, 2016.

25. Lau WY and Lai EC: The current role of radiofrequency ablation in the management of hepatocellular carcinoma: A systematic review. Ann Surg 249: 20-25, 2009.

26. Poggi G, Tosoratti N, Montagna B and Picchi C: Microwave ablation of hepatocellular carcinoma. World J Hepatol 7: 2578-2589, 2015.

27. Wright AS, Sampson LA, Warner TF, Mahvi DM and Lee FT Jr: Radiofrequency versus microwave ablation in a hepatic porcine model. Radiology 236: 132-139, 2005.

28. Lucchina N, Tsetis D, Ierardi AM, Giorlando F, Macchi E, Kehagias E, Duka E, Fontana F, Livraghi L and Carrafiello G: Current role of microwave ablation in the treatment of small hepatocellular carcinomas. Ann Gastroenterol 29: 460-465, 2016.

29. Wang JH, Wang CC, Hung CH, Chen CL and Lu SN: Survival comparison between surgical resection and radiofrequency ablation for patients in BCLC very early/early stage hepatocellular carcinoma. J Hepatol 56: 412-418, 2012.

30. Harada N, Shirabe K, Maeda T, Kayashima H, Takaki S and Maehara Y: Comparison of the outcomes of patients with hepatocellular carcinoma and portal hypertension after liver resection versus radiofrequency ablation. World J Surg 40: 1709-1719, 2016.

31. Kim SJ, Lee KK and Kim DG: Tumor size predicts the biological behavior and influence of operative modalities in hepatocellular carcinoma. Hepatogastroenterology 57: 121-126, 2010.

32. Chen X, Zhang B, Yin X, Ren Z, Qiu S and Zhou J: Lipiodolized transarterial chemoembolization in hepatocellular carcinoma patients after curative resection. J Cancer Res Clin Oncol 139: 773-781, 2013.

33. Lazzara C, Navarra G, Lazzara S, Barbera A, Saitta C, Raimondo G, Latteri S and Curro G: Does the margin width influence recurrence rate in liver surgery for hepatocellular carcinoma smaller than $5 \mathrm{~cm}$ ? Eur Rev Med Pharmacol Sci 21: 523-529, 2017.

34. Qian GJ, Wang N, Shen Q, Sheng YH, Zhao JQ, Kuang M, Liu GJ and Wu MC: Efficacy of microwave versus radiofrequency ablation for treatment of small hepatocellular carcinoma: Experimental and clinical studies. Eur Radiol 22: 1983-1990, 2012.

35. Huo TI, Liu WY, Wu JC, Huang YH, King KL, Loong CC, Lee PC, Chang FY and Lee SD: Deterioration of hepatic functional reserve in patient with hepatocellular carcinoma after resection: Incidence risk factors, and association with intrahepatic tumor recurrence. World J Surg 28: 258-262, 2004.

36. Yang W: Current status and prospective of imaging guided radiofrequency ablation in medium to large sized hepatocellular carcinomas. World Chin J Digestol 30: 4771, 2015 (In Chinese). 
37. Pompili M, Saviano A, de Matthaeis N, Cucchetti A, Ardito F, Federico B, Brunello F, Pinna AD, Giorgio A, Giulini SM, et al Long-term effectiveness of resection and radiofrequency ablation for single hepatocellular carcinoma $\leq 3 \mathrm{~cm}$. Results of a multicenter Italian survey. J Hepatol 59: 89-97, 2013.

38. Zhang P, Gu A and Shi DM: Analysis of the status of postoperative recurrence and metastasis of hepatocellular carcinoma and its influencing factors. Oncol Prog 4, 2017.

39. Li T, Wang SK, Zhou J, Sun HC, Qiu SJ, Ye QH, Wang L and Fan J: Positive HBcAb is associated with higher risk of early recurrence and poorer survival after curative resection of HBV-related HCC. Liver Int 36: 284-292, 2016.
40. Huang JQ, Peng MH, Zou YQ, Yang DH, Chen B and Xiao KY: Analysis of risk factors for early recurrence of primary hepatocellular carcinoma after radical hepatectomy. Chinese J Practl Surg 5: 418-420, 2009 (In Chinese).

(i) (3) () This work is licensed under a Creative Commons Attribution-NonCommercial-NoDerivatives 4.0 International (CC BY-NC-ND 4.0) License. 